\title{
Subcellular fractionation and molecular characterization of the pellicle and plasmalemma of Neospora caninum
}

\author{
Y. LEI ${ }^{1}$, D. BIRCH ${ }^{2}$, M. DAVEY ${ }^{1}$ and J. T. ELLIS ${ }^{1 *}$ \\ ${ }^{1}$ Department of Cell and Molecular Biology, Faculty of Science, University of Technology, Sydney, Gore Hill, \\ NSW 2065 Australia \\ ${ }^{2}$ Microscopy Unit, Department of Biological Sciences, University of Macquarie, North Ryde, NSW 2131 Australia
}

(Received 21 Fanuary 2005; revised 4 April 2005; accepted 7 April 2005)

\section{SUMMARY}

A characteristic structural feature of Toxoplasma gondii and Neospora caninum is the presence of a triple-membrane pellicle, on the zoite stages of their complex life-cycle. Here we report the results of electron microscopic studies which show that the pellicle is made of a typical plasmalemma covered on its cytoplasmic side by a system of flattened vesicles named the inner membrane complex. Using methods described previously for the purification of pellicle and plasmalemma fractions from T. gondii, we have evaluated the same methodology for the preparation of pellicles and plasmalemma from N. caninum. The approach used involved subcellular fractionation and sucrose gradient centrifugation to prepare fractions containing pellicles. Plasmalemma was prepared by extraction of this fraction with a high salt glycerol treatment. Fractions containing membrane structures were identified by electron microscopy, and the proteins and antigens present in them were subsequently studied by SDS-PAGE and Western blotting. Electron microscopy of the pellicle fractions of N. caninum demonstrated preservation of the triple-membrane structure which is identical to that found in T. gondii. SDS-PAGE of the pellicle fractions revealed it contained several major proteins. Analyses revealed that the plasmalemma of $N$. caninum contained 2 abundant proteins in addition to other much lower abundance antigens detectable by monoclonal antibodies. These studies therefore report, for the first time, a detailed molecular characterization of the pellicle and plasmalemma of N. caninum.

Key words: Neospora caninum, Toxoplasma gondii, pellicle, plasmalemma, surface antigen, subcellular fractionation.

\section{INTRODUCTION}

Toxoplasma gondii and Neospora caninum are two closely related apicomplexan parasites. A unique feature of $T$. gondii and $N$. caninum tachyzoites is the presence of a complex pellicle containing 3 distinct peripheral membranes (Ogino and Yoneda, 1966). Two closely opposed membranes called the inner membrane complex (IMC) arise at the anterior polar ring and terminate at the posterior ring (Sheffield and Melton, 1968; Sulzer et al. 1974). A layer of microtubules is localized under the inner membrane complex. These microtubules originate from the inner ring of the conoid and project toward the posterior but do not reach the posterior portion of the cell. An outer plasma membrane, a third membrane also known as the plasmalemma, is in contact with the external environment. This encloses the entire organism and was considered analogous to a typical cell membrane (Vivier et al. 1970). The function of the pellicle has not been determined, but its close association with the microtubules suggests that it

* Corresponding author: Department of Cell and Molecular Biology, Faculty of Science, University of Technology, Sydney, Gore Hill, NSW 2065 Australia. Tel: +612 9514 4161. Fax: +612 9514 4026. E-mail: john.ellis@uts.edu.au might be involved in maintaining structural integrity and the gliding motility of the cell. In addition to any involvement in cell motility, the pellicles of $T$. gondii and $N$. caninum must play an important role in host cell attachment and invasion, which is a prerequisite for their survival and proliferation.

Although the $T$. gondii pellicle has been well described by conventional transmission electron microscopy and freeze-fracture studies (Vivier and Petitpres, 1969; Porchet and Torpier, 1977; Tryon, 1979; Rabjeau et al. 1997), only a few studies have tried to isolate the pellicle (Vivier and Petitpres, 1969; Porchet and Torpier, 1977; Tryon, 1979; Foussard et al. 1990). Rabjeau et al. (1997) also described a method to isolate plasmalemma-enriched fractions of $T$. gondii and investigated their protein composition. Despite the intense effort put into research on the most abundant surface antigens of T. gondii (Handman, Goding and Remington, 1980; Couveur et al. 1988) known as SRS (SAG1-related sequences) proteins (Boothroyd et al. 1998; Jung, Lee and Grigg, 2004), few attempts have been made to determine the protein composition of the pellicle and plasmalemma directly (Foussard et al. 1990; Rabjeau et al. 1997). The main impediment for the isolation of these subcellular fractions is the need for large numbers of tachyzoites for the purification process. Consequently, most previous studies on 
plasma membranes of $T$. gondii and $N$. caninum have generally used Triton extraction or enrichment along with the generation of monoclonal antibodies (MAB) (Nichols and Chiappino, 1987, Morrissette et al. 1994; Hemphill, 1996; Hemphill and Gottstein, 1996; Hemphill et al. 1997 a, b. Howe et al. 1998, Sonda et al. 1998, Björkman and Hemphill, 1998, Schares et al. 1999, Mann \& Beckers, 2001). These approaches do not provide complete information on the membrane composition or structure.

In order to investigate the structure and protein composition of pellicles and plasmalemma of $N$. caninum, we implemented isolation methods developed for $T$. gondii and applied them to $N$. caninum. The aims of this study were to (1) evaluate methods for the purification of pellicles and plasmalemma, (2) identify the main proteins and antigens in fractions enriched for them, and (3) identify any molecular differences which exist in pellicle and plasmalemma between the two species.

\section{MATERIALS AND METHODS}

\section{Cell culture and parasites}

Tachyzoites of $T$. gondii $\mathrm{RH}$ strain and $N$. caninum $\mathrm{Nc}-1$ strain were grown in $250 \mathrm{~cm}^{2}$ tissue culture flasks by continuous passage in Vero cell monolayers using RPMI 1640 media supplemented with $2 \%$ newborn bovine serum (NBS), $2 \mathrm{~mm}$ L-glutamine, $50 \mathrm{U} / \mathrm{ml}$ penicillin and $50 \mu \mathrm{g} / \mathrm{ml}$ streptomycin at $37{ }^{\circ} \mathrm{C}$ in a $5 \% \mathrm{CO}_{2}$ atmosphere. Fresh tachyzoites of $\mathrm{RH}$ and $\mathrm{Nc}-1$ were harvested from infected Vero cells typically on day $5(\mathrm{RH})$ or day $8(\mathrm{Nc}-1)$ postinfection.

\section{Antibodies}

The following antibodies were used in these studies. 5.2.15 is a monoclonal antibody (MAB) which recognizes a $38 \mathrm{kDa}$ tachyzoite surface antigen of $N$. caninum (Schares et al. 1999); MAB 4.7.12 recognizes a $19 \mathrm{kDa}$ surface antigen of $N$. caninum (Schares et al. 1999); MAB Ncmab-4 recognizes NcSAG1 (Björkman and Hemphill, 1998) while MAB DG52 (kindly provided by Professor John Boothroyd, Stanford University) detects the T.gondii surface protein SAG1. A mouse polyclonal antiserum, raised by infection of Qs mice with $N$. caninum tachyzoites (Nc-Liverpool) was kindly provided by Dr H. Quinn. A goat anti-mouse IgG alkaline phasphatase-conjugated secondary antibody was used at a dilution of $1: 1000$ (Sigma).

\section{Tachyzoite purification}

Tachyzoites were harvested from cell cultures by first gently scraping the monolayer contents into $10 \mathrm{ml}$ of cold PBS (containing both host cells, debris and tachyzoites in suspension), and then passing the suspension 3 times through a 26-gauge needle to release tachyzoites from host cells. These were then purified free from host cell debris by filtering them through a $3 \cdot 0 \mu \mathrm{m}$ filter membrane (Morrissette et al. 1994) leaving essentially tachyzoites only in solution. This process was monitored by light microscopy and repeated if necessary. The purified tachyzoites were washed in cold PBS buffer by 3 centrifugations at $1000 \mathrm{~g}$ for $20 \mathrm{~min}$ at $4{ }^{\circ} \mathrm{C}$. They were then resuspended into $2 \mathrm{ml}$ of homogenization buffer (HB, $250 \mathrm{~mm}$ sucrose, $1 \mathrm{~mm}$ EDTA, $5 \mathrm{~mm}$ triethanolamine-HCl, $\mathrm{pH} 7 \cdot 5$ ) at a concentration of $1 \times 10^{9}$ parasites $/ \mathrm{ml}$ and then immediately used for pellicle isolation. All subsequent operations were performed on ice or at $4{ }^{\circ} \mathrm{C}$.

\section{Pellicle isolation}

The methodology of Foussard et al. (1990) and Rabjeau et al. (1997) was used in the following way. A flow diagram of the process is shown in Fig. 1.

\section{Tachyzoite disruption}

Cell disruption was performed by sonication in a cell ultrasonic processor as described previously (Foussard et al. 1990; Rabjeau et al. 1997). Briefly, $2 \mathrm{ml}$ of purified tachyzoite suspension containing $2 \times 10^{9}$ parasites in a $10 \mathrm{ml}$ vol. tube were placed in an ice-water bath and sonicated 3 times for $15 \mathrm{~s}$ at $20 \mathrm{mHz}$. To assist in the purification, $1 \mathrm{mg} / \mathrm{ml}$ DNase (Deoxyribounclease 1, Sigma) and a cocktail of proteinase inhibitors containing $1 \mu \mathrm{M}$ leupeptin, $2 \mathrm{~mm}$ phenylmethysulfonyl fluoride, $2 \mu \mathrm{M}$ aprotinin, $3 \mu \mathrm{M}$ pepstatin A (Sigma) were added to the preparation. The sonicator tip was placed so that it reached half way up the suspension level. The Ultrasonic Homogenizer 4710 was set at $20 \%$ of its maximal power for $15 \mathrm{sec}$ and the sample was pulsed 3 times. The sample was allowed to cool at $4{ }^{\circ} \mathrm{C}$ for $2 \mathrm{~min}$ in between pulses.

After sonication, the parasites were centrifuged to remove unbroken tachyzoites and nuclei at $600 \boldsymbol{g}$ for $5 \mathrm{~min}$; the pellet was then resuspended in $2 \mathrm{ml}$ of $\mathrm{HB}$ and the sonication was repeated under the same conditions. After the low-speed centrifugation, the supernatants from each step $\left(\mathrm{S}_{1}\right.$ and $\left.\mathrm{S}_{2}\right)$ were pooled and used for pellicle purification.

\section{Subcellular fractionation}

The supernatants $S_{1}$ and $S_{2}$ were pooled and centrifuged at $30000 \boldsymbol{g}$ for $30 \mathrm{~min}$ in an SW rotor of a Sorvall Super T-21 high-speed centrifuge. Purification of pellicles from the pellet was immediately accomplished by 4-step discontinuous sucrose density-gradient centrifugation, as described below. 


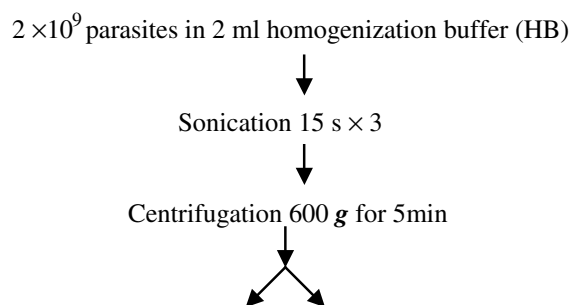

Pellet in $2 \mathrm{ml} \mathrm{HB} \quad$ Supernatant $\left(\mathrm{S}_{1}\right)$

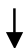

Centrifugation $600 \mathrm{~g}$ for $5 \mathrm{~min}$

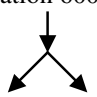

Pellet (discard) Supernatant $\left(\mathrm{S}_{2}\right)$

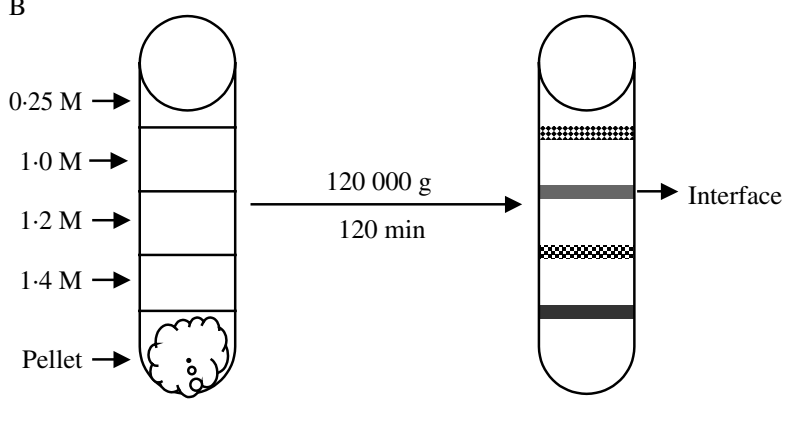

Fig. 1. Diagrams showing the procedure used to prepare pellicles and plasmalemma of Toxoplasma gondii and Neospora caninum. (A) diagrammatic representation of the methods used; and (B) purification of pellicle by discontinuous sucrose gradient ultracentrifugation. Sample was deposited at the bottom of a discontinuous sucrose gradient (containing the following steps: $1 \cdot 6 \mathrm{M}$ : $3.03 \mathrm{ml}, 1.4 \mathrm{M}: 2.42 \mathrm{ml}, 1.2 \mathrm{M}: 2.42 \mathrm{ml}, 1 \cdot 0 \mathrm{M}: 2.42 \mathrm{ml}$, $0.25 \mathrm{M}: 1.21 \mathrm{ml}$ ) that was then centrifuged at $120000 \mathrm{~g}$ for $2 \mathrm{~h}$ in a DT54 rotor. After the 4-step sucrose gradient ultracentrifugation, the pellicle fraction was recovered at the $1 \cdot 0-1 \cdot 2 \mathrm{M}$ interface.

Purification of pellicle by 4-step discontinuous sucrosegradient ultracentrifugation. The pellet from the $30000 \boldsymbol{g}$ spin was resuspended in $10 \mathrm{~mm}$ Tris- $\mathrm{HCl}$ buffer (pH 7.5) and transferred into a $10 \mathrm{ml}$ vol. tube. The suspension was then adjusted to $1.6 \mathrm{M}$ sucrose with $2.0 \mathrm{M}$ sucrose (by adding $2.42 \mathrm{ml}$ of $2.0 \mathrm{M}$ sucrose for every $0.606 \mathrm{ml}$ of suspension). This was deposited at the bottom of a 4 -step sucrose gradient $(1.6 \mathrm{M}: 3.03 \mathrm{ml}$ sample, $1.4 \mathrm{M}: 2.42 \mathrm{ml}, 1.2 \mathrm{M}$ : $2.42 \mathrm{ml}, 1 \cdot 0 \mathrm{M}: 2.42 \mathrm{ml}, 0.25 \mathrm{M}: 1.21 \mathrm{ml}$, total $11.5 \mathrm{ml}$ ) which was centrifuged for $2 \mathrm{~h}$ at $120000 \mathrm{~g}$ in an ultra-speed Sorvall OTD 55B rotor (Fig. 1B). The material at the $1 \cdot 0-1 \cdot 2 \mathrm{M}$ interface was collected using a butterfly needle connected to a syringe, diluted with 3 volumes of $\mathrm{HB}$ and concentrated by centrifugation at $30000 \mathrm{~g}$ for $30 \mathrm{~min}$. The pellet was immediately processed for electron microscopy, preparation of plasmalemma or frozen at $-80{ }^{\circ} \mathrm{C}$ for immunoblot analysis.

Plasmalemma isolation. Attempts to purify plasmalemma were performed using the following procedure that involves dissociation, freezing and thawing, sonication and ultracentrifugation. Enriched pellicle preparations $(4 \mathrm{mg}$ ) were incubated at $4{ }^{\circ} \mathrm{C}$ for $2 \mathrm{~h}$ on an orbital wheel in $4 \mathrm{ml}$ of dissociation buffer containing $10 \mathrm{~mm}$ Tris-HCl, pH 7.2, $0 \cdot 5 \mathrm{M}$ $\mathrm{NaCl}, 5 \%$ glycerol and $0.02 \%$ DNase (Deoxyribounclease 1, Sigma). The preparation was then frozen in liquid nitrogen, thawed in a $25{ }^{\circ} \mathrm{C}$ water bath once, and then disrupted by sonication within an Ultrasonic Homogenizer with a $1 \mathrm{~cm}$ tip for $3 \times 10 \mathrm{sec}$. The suspension was centrifuged at $10000 \boldsymbol{g}$ to sediment components that were not disrupted. The supernatant was then adjusted for ultracentrifugation as follows. After spinning at $10000 \boldsymbol{g}$ to sediment components that were not disrupted, the supernatant was adjusted to $0 \cdot 7 \mathrm{M}$ sucrose with $2 \mathrm{M}$ sucrose and purification of the plasmalemma was achieved by ultracentrifugation at $140000 \mathrm{~g}$ for $15 \mathrm{~h}$ at $4{ }^{\circ} \mathrm{C}$. After ultracentrifugation, the gradient was collected using a butterfly needle connected to a syringe as $12 \times 0.5 \mathrm{ml}$ fractions covering the bottom to the top of the gradient; the pellet was then resuspended in $10 \mathrm{~mm}$ Tris- $\mathrm{HCl}$ buffer. These different samples were processed for electron microscopy, SDS-PAGE and immunocharacterization by Western blotting.

\section{Sodium dodecyl sulphate-polyacrylamide gel electrophoresis (SDS-PAGE) of pellicle and plasmalemma}

Protein concentration of samples was determined by the Bio-Rad DC Protein method with bovine serum albumin (BSA) as a standard. SDS-PAGE under non-reducing or reducing conditions was performed on $12 \%$ separation gels in order to identify proteins present in the pellicle and plasmalemma fractions. Membrane proteins were solubilized in sample buffer (reducing condition: $2 \cdot 3 \% \mathrm{SDS}, 5 \% \beta$-mercaptoethanol, $10 \%$ glycerol, $0 \cdot 5 \%$ bromophenol blue, $62 \mathrm{~mm}$ Tris-HCI pH 6.8; non-reducing condition: the same buffer without $\beta$-mercaptoethanol) by heating at $100{ }^{\circ} \mathrm{C}$ for $3 \mathrm{~min}$. Molecular weight markers (Bio-Rad) were also run in order to estimate protein sizes. Equal amounts of proteins from each experimental sample were separated by electrophoresis on SDS-PAGE gels. Proteins were then visualized by staining in Coomassie Blue $(0 \cdot 2 \%$ Coomassie Brilliant Blue R (Sigma), 50\% methanol, 10\% glacial acetic acid) for $30 \mathrm{~min}$ followed by destaining (46\% methanol, $10 \%$ glacial acetic acid), or silver staining using a commercial kit (Amersham Pharmacia Biotech AB, Uppsala Sweden). Alternatively, proteins were transferred onto polyvinylidene difluoride membrane (PVDF, Millipore) as described below, by Western blotting. 


\section{Western blot analysis}

After SDS-PAGE, proteins from the different groups were electrophoretically transferred onto PVDF sheets in Tris-glycine transfer buffer $(25 \mathrm{mM}$ Tris-base, $200 \mathrm{~mm}$ glycine and $20 \%$ methanol) for $2 \mathrm{~h}$ at $100 \mathrm{~V}$ (Harlow and Lane, 1988). Non-specific binding sites on the membranes were blocked in $5 \%$ skim milk powder in PBS (blocking solution) for $16 \mathrm{~h}$ at $4{ }^{\circ} \mathrm{C}$. After 3 washes in PBS containing $0.03 \%$ Tween-20 (Tween-20/PBS) over $30 \mathrm{~min}$, the membranes were incubated in the presence of primary antibodies (1:50 for MAB 5.2.15, 1:10 for 4.7.12 and 1:500 for DG52) in blocking solution for $2 \mathrm{~h}$. The membrane was left at room temperature with constant agitation, prior to washing with 3 changes of $0.03 \%$ Tween-20/PBS over $30 \mathrm{~min}$. The membranes were then probed with goat anti-mouse IgG alkaline phosphatase conjugate in blocking solution (1:1000; Sigma) for $1 \mathrm{~h}$. The membranes were washed 3 times in PBS and developed in the presence of 5-bromo-4-chloro-3-indolyl phosphate and nitroblue tetrazolium (Sigma).

\section{Transmission electron microscopy}

For standard transmission electron microscopy, subcellular fractions were immediately fixed for $2 \mathrm{~h}$ in $4 \%$ paraformaldehyde and $3 \%$ glutaraldehyde in $0 \cdot 1$ м phosphate buffer $(\mathrm{pH} 7 \cdot 2)$ at $4{ }^{\circ} \mathrm{C}$. Following 3 washes in $0.1 \mathrm{M}$ phosphate buffer the fractions were post-fixed in $2 \% \mathrm{OsO}_{4}$ in the same buffer for $60 \mathrm{~min}$ at room temperature. The fractions were then dehydrated in a graded series of ethanol $(50 \%, 75 \%$, $95 \%$ and $100 \%$ ) for $15 \mathrm{~min}$ each at $-20{ }^{\circ} \mathrm{C}$, infiltrated in $1: 1(\mathrm{v}: \mathrm{v})$ LR White Resin : $100 \%$ ethanol at $-20{ }^{\circ} \mathrm{C}$ for $1 \mathrm{~h}$. After a further $1 \mathrm{~h}$ of infiltration with LR White Resin, the fractions were embedded in gelatine capsules and polymerized overnight at $65{ }^{\circ} \mathrm{C}$. Ultrathin sections were prepared using an ultramicrotome (Ultracut/Leica) with a diamond knife (Druker) and loaded onto 300 mesh, Pioloform coated copper grids (ProSciTech). The sections were stained with $2 \%$ aqueous uranyl acetate and Reynold's lead citrate for $4 \mathrm{~min}$. Finally, sections were examined with a Philips CM 10 transmission electron microscope.

Negative staining was used to identify the contents of the plasmalemma fractions. The fractions were diluted 1:1 in PBS and allowed to settle onto plasticcoated, copper grids for $1 \mathrm{~min}$. Excess liquid was removed and the grids were then negatively stained with $2 \%$ aqueous uranyl acetate $(\mathrm{pH} 7 \cdot 2)$ for $1 \mathrm{~min}$ and examined with a Philips CM 10 transmission electron microscope.

\section{RESULTS}

Preparation of pellicle and plasmalemma fractions was performed using principles and methods described by Foussard et al. (1990), and Rabjeau et al. (1997). Electron microscopy of ultrathin sections was used to assess the purity and contents of the fractions. Ultrastructurally, tachyzoites of $T$. gondii and $N$. caninum are very similar morphologically. They possess a 3-layered membrane pellicle consisting of an outer plasmalemma and 2 closely applied membranes that form an inner membrane complex. Their apical end is composed of microtubles, apical rings, conoid, and a polar ring (Fig. 2).

\section{Analysis of subcellular fractions}

Sucrose density-gradient centrifugation of insoluble membrane preparations of $T$. gondii and $N$. caninum tachyzoites resulted in 4 distinct bands, which were localized at different interfaces of the gradient. Pellicles were found, by electron microscopy, among the rapidly sedimented material, which floated to the 1-1.2 M interface when subjected to gradient purification. Transmission electron micrographs of ultrathin sections derived from the $1-1 \cdot 2 \mathrm{M}$ fractions of $T$. gondii and $N$. caninum revealed that the pure pellicle fragments were heterogeneous in size with a characteristic triple-membrane pellicle structure consisting of an inner membrane complex, lying just beneath the plasmalemma (Fig. 3). Higher magnification confirmed the presence of the inner membrane complex, with its associated subpellicular microtubules (Fig. 3 bottom panels). Single small membranes that could have originated from either damaged pellicles or intracytoplasmic membranes were also sometimes seen. However, the preservation of the subpellicular microtubules in most of the single membranes indicates that the single membranes had been part of the pellicles.

Enrichment for plasmalemma was achieved by high salt-glycerol treatment followed by sonication of the $1-1 \cdot 2 \mathrm{M}$ pellicle-enriched fraction. Negative staining of plasmalemma fractions that were examined by electron microscopy showed membranous vesicles of sizes ranging from 50 to $100 \mathrm{~nm}$ for both species (Fig. 4). No apparent morphological or size differences were observed in these between RH and Nc-1. Transmission electron microscopy of these vesicles showed a typical single membrane structure. These vesicles were present throughout the gradient, and there was no obvious enrichment for them by the gradient. In addition, Western blotting of gradient fractions derived from $T$. gondii with the MAB DG52 (which detects SAG1) showed this was present in all gradient fractions tested for T. gondii (data not shown).

\section{Analysis of the composition of the purified pellicle fractions}

Proteins present in the pellicle fractions $(1 \cdot 0-1 \cdot 2 \mathrm{M}$ interface) of the two species were examined by 


\section{T. gondii}

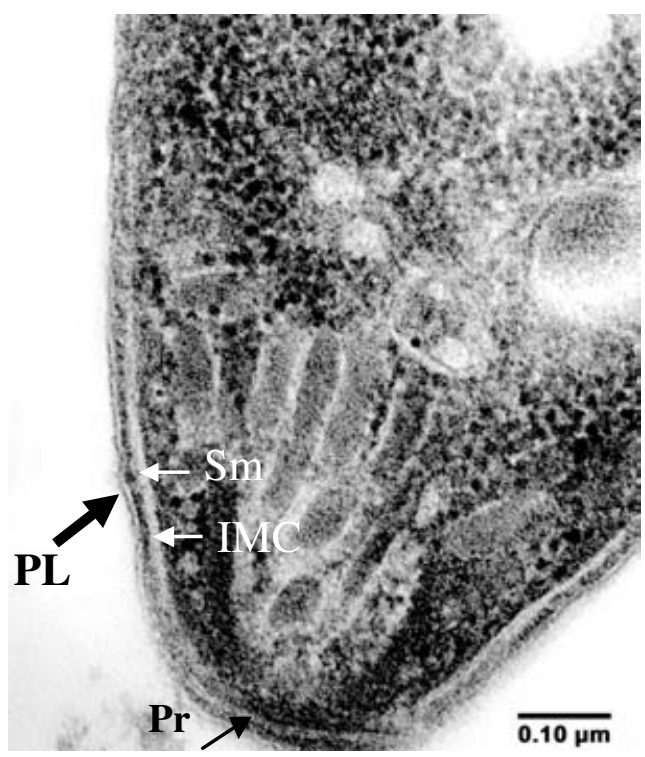

N. caninum

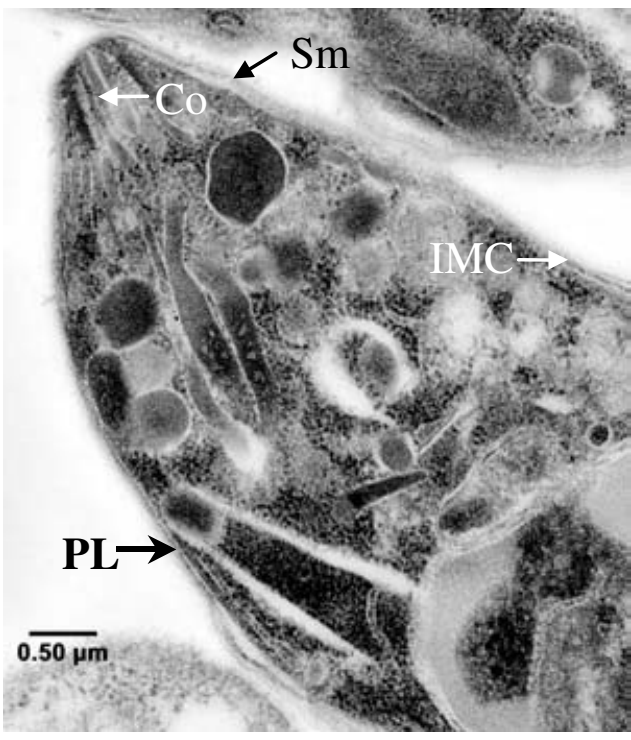

Fig. 2. Transmission electron micrographs of the anterior end of tachyzoites of Toxoplasma gondii and Neospora caninum. Thick black arrows denote the outer plasmalemma and thin white arrows denote the inner membrane complex, which begins at the apical polar ring and runs in close apposition to the plasmalemma along the entire length of the parasite, subcellular microtubules and polar rings. PL, plasmalemma; IMC, inner membrane complex; Pr, polar ring; Sm, subcellular microtubule, Co, conoid.

\section{T. gondii}
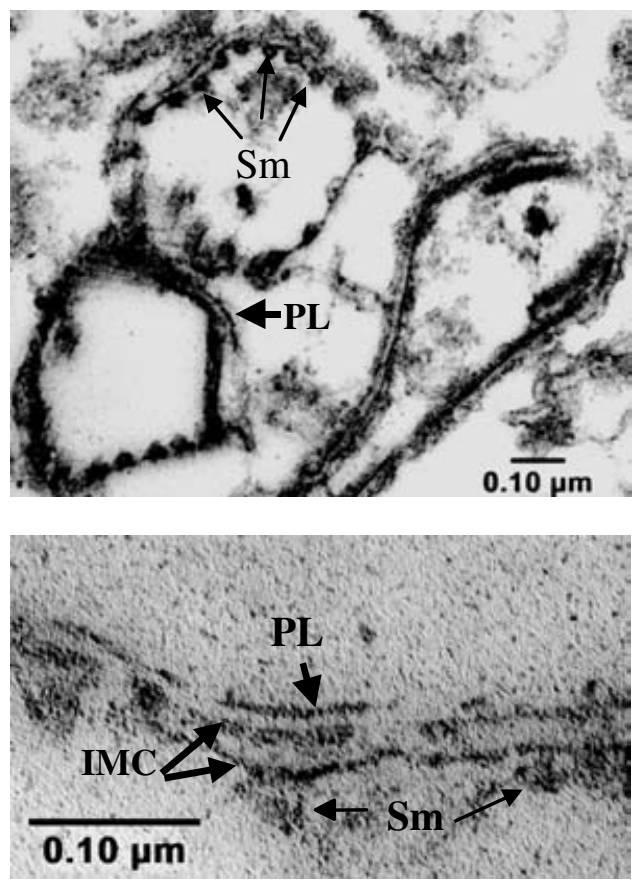

\section{N. caninum}
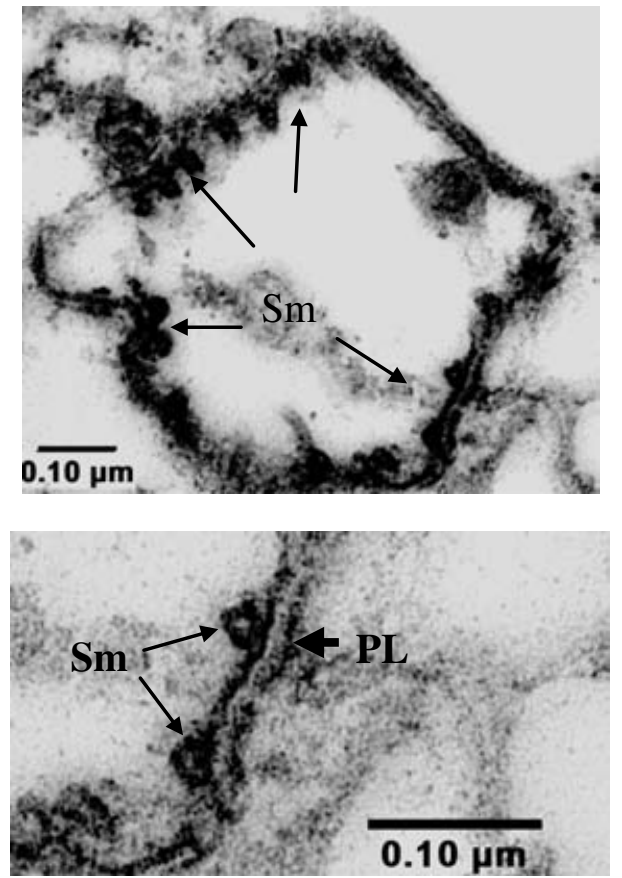

Fig. 3. Electron micrographs of pellicle and plasmalemma preparations from Toxoplasma gondii and Neospora caninum Transmission electron micrographs of the pellicle fraction from the $1 \cdot 0-1 \cdot 2 \mathrm{M}$ interface of the gradient, which almost exclusively contains pellicles. Subcellular microtubules $(\mathrm{Sm})$ remain associated with the inner membrane complex (IMC). PL, plasmalemma. A higher magnification of the pellicle fraction (botton panels) shows the typical triple-layer membrane structure composing of an outer PL and an IMC).

SDS-PAGE and compared with a whole-cell extract. Coomassie Blue staining of SDS-PAGE gels of the purified pellicle fraction from $T$. gondii showed 2 major proteins at 30 and $43 \mathrm{kDa}$ respectively and several other, less prominent, proteins at $22,30,43$, 50, 55, 79, 90 and $160 \mathrm{kDa}$ respectively (Fig. 5). 


\section{T. gondii}

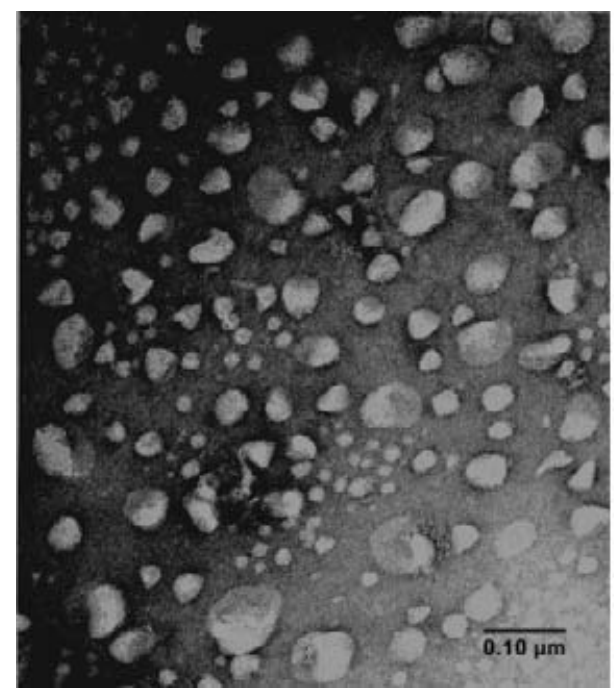

N. caninum

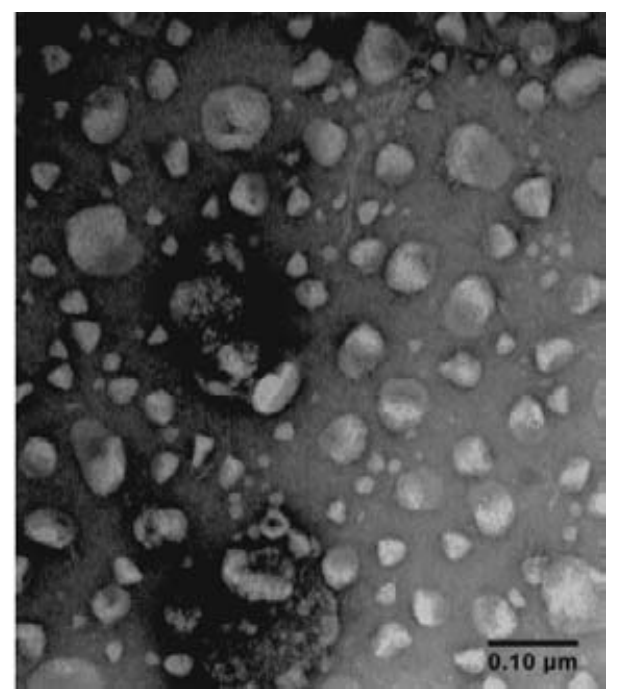

Fig. 4. Transmission electron microscopy of a pellicle fraction treated with high salt glycerol dissociation buffer, freezing/thawing and sonication showing a number of uni- and plurimembranous vesicles of heterogenous size. Negatively stained specimen.

SDS-PAGE analysis of the pellicle fraction from N. caninum also showed a complex protein profile, dominated by 5 major bands in the 29, 40, 50, 65 and $110 \mathrm{kDa}$ ranges (Fig. 6). Other proteins, present in lower but significant amounts were found at 32,38 , 55,70 and $150 \mathrm{kDa}$.

\section{Analysis of the purified plasmalemma fractions}

SDS-PAGE of the purified plasmalemma fractions showed a different protein profile compared to the pellicle. In contrast to the complex profile obtained from the pellicle fractions, only 2 abundant proteins (approx. 30 and $22 \mathrm{kDa}$ respectively) were detected upon electrophoresis of plasmalemma fractions from either $T$. gondii or $N$. caninum (Fig. 5) by silver staining. In some gels stained with Coomassie Blue the apparent mobility of these proteins was 32 and $25 \mathrm{kDa}$ respectively (see Fig. 6 for an example). The sizes of these 2 bands did not change when the protein electrophoresis was performed under either reducing or non-reducing conditions (not shown).

Following SDS-PAGE, fractions containing plasmalemma from both species were investigated by Western blotting using monoclonal antibodies that recognize specific $T$. gondii and $N$. caninum surface molecules respectively. MAB DG 52 reacted under denaturing conditions very intensely with SAG1 (P30) (Fig. 5), the major surface antigen of T. gondii Similarly, probing of plasmalemma fractions from $T$. gondii with a polyclonal serum to SAG2 resulted in the detection of a $22 \mathrm{kDa}$ antigen which co-migrated with the abundant $22 \mathrm{kDa}$ protein identified in plasmalemma fractions by protein staining methodologies (Fig. 5). This antibody also showed some minor cross-reactivity to the $30 \mathrm{kDa}$ SAG1 antigen.

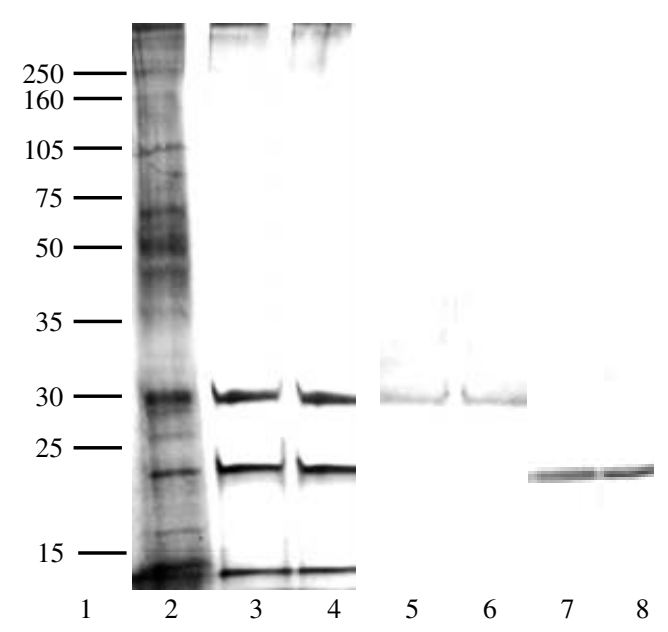

Fig. 5. SDS-PAGE and Westen blotting of Toxoplasma gondii $\mathrm{RH}$ pellicle and plasmalemma fractions with $\mathrm{MAB}$ DG52 (anti- $T$. gondii-SAG1) or anti- $T$. gondii-SAG2 antibodies. Lane 1, molecular weight marker; lane 2, RH pellicle fraction separated by SDS-PAGE and stained with Commassie Blue; lane 3, RH plasmalemma fractions 11 and 12 stained with Commassie Blue (lane 4); lane 5, Western blotting of plasmalemma fractions 11 and 12 (lane 6) with anti-SAG1; lane 7, Western blotting of plasmalemma fractions 11 and 12 (lane 8) with antiSAG2.

We therefore conclude that the 2 abundant proteins identified in the $T$. gondii plasmalemma were SAG1 and SAG2.

For N. caninum, when Western blots of nonreduced plasmalemma were probed with MABs 5.2.15 and 4.7.12, both of which are known to detect surface antigens of $N$. caninum (Schares et al. 1999), MAB 5.2.15 reacted strongly with an antigen of $38 \mathrm{kDa}$ (Fig. 6, lane 3); whereas MAB 4.7.12 reacted 


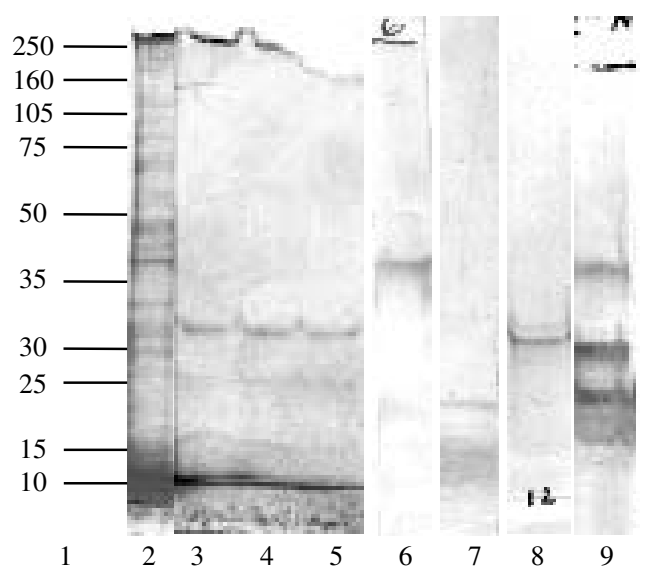

Fig. 6. SDS-PAGE and Western blot analyses of pellicle and plasmalemma fractions from Neospora caninum $\mathrm{Nc}-1$ isolate. Lane 1, position of molecular weight markers; lane 2, Coomassie Blue-stained pellicle fraction run under reducing conditions; lanes $3-5$, Coomasie Bluestained plasmalemma fractions 12,11 and 10 respectively run under reducing conditions; lane 6 , Western blotting of plasmalemma fraction 12 with MAB 5.2.15 under nonreducing conditions; lane 7 , Western blotting of the plasmalemma fraction 12 with monoclonal antibody 4.7.12 under reducing conditions; lane 8 , Western blotting of the plasmalemma fraction 12 with NcmAb-4 under reducing conditions; and lane 9, Western blotting of the plasmalemma fraction 12 (under reducing conditions) with mouse anti N. caninum (Nc-Liverpool) serum. The positions of the molecular weight markers are shown.

with the $38 \mathrm{kDa}$ antigen and another antigen of $10 \mathrm{kDa}$ (not shown). When reduced plasmalemma protein was probed with MABs 5.2.15 and 4.7.12, MAB 5.2.15 failed to recognize any antigens whereas MAB 4.7.12 recognized an antigen of $19 \mathrm{kDa}$ and several others in a smear in the $10-15 \mathrm{kDa}$ range (Fig. 6, lane 7). Ncmab-4 detected the abundant 30/ $32 \mathrm{kDa}$ antigen in pellicle and plasmalemma preparations (Fig. 6, lane 8), whereas a mouse serum raised by infection with $N$. caninum detected 5 antigens in the plasmalemma fractions by Western blotting (Fig. 6, lane 9), including the 38, 30, $22 \mathrm{kDa}$ surface antigens studied here. These results therefore provide additional substantial evidence that the membrane vesicles obtained from $N$. caninum contained plasmalemma since they contained known surface antigens.

\section{I S CUSSION}

Few studies describing the isolation and biochemical analysis of the $T$. gondii pellicle (Mauras, Laget and Sennety, 1977; Tryon 1979; Foussard et al. 1990) and plasmalemma (Rabjeau et al. 1997) are available. No data regarding the isolation of the $N$. caninum pellicle and plasmalemma have been published, although analyses of detergent extracts and cell localization studies with a number of monoclonal antibodies have identified the presence of several antigens (e.g. Hemphill et al. 1997a, b; Björkman and Hemphill, 1998; Schares et al. 1999). The major reasons for this are probably associated with the difficulty in obtaining large numbers of tachyzoites of $T$. gondii and N. caninum; the fact that these organisms are relatively resistant to the classical methods of cell disruption (in these protozoa a layer of subcellular microtubules renders the cells more resistant to rupture); and lack of well-defined markers like monoclonal antibodies raised specially to the pellicles of these parasites with which to characterize the isolated structures.

The present study was undertaken to evaluate whether methods previously described for the study of subcellular fractions of $T$. gondii could also be used for the study of $N$. caninum. Hence we attempted to isolate and characterize the pellicles and plasmalemma fractions from tachyzoites of both $T$. gondii and $N$. caninum so that the composition of their membrane structures could be compared. The present study is therefore the first to describe attempts to physically isolate and characterize the pellicle and plasmalemma from $N$. caninum.

The procedures used in this study were described previously by Foussard et al. (1990) and Rabjeau et al. (1997), in which the pellicle and plasmalemma of $T$. gondii were isolated and characterized. The method is based on subcellular fractionation, which was used to obtain a pure pellicle fraction. After disrupting parasites by sonication, the lysate was subjected to differential centrifugation. Finally a 4-step discontinuous sucrose gradient ultracentrifugation was used to purify the pellicle fraction, which was found at the 1-1.2 M sucrose interface of the gradient. Electron microscopy showed that the 1-1.2 m fraction was highly enriched for the pellicle, which contained the outer plasmalemma, the inner membrane complex and the subpellicular microtubules. The SDS-PAGE protein profile obtained from the purified pellicle from $T$. gondii is very similar to the results obtained by Foussard et al. (1990) who reported several proteins at 30, 43, 50 and $79 \mathrm{kDa}$. Western blotting also showed this fraction was enriched for SAG 1. Hence in these studies we were therefore able to reproduce the results of Foussard et al. (1990) thereby providing confidence in the subcellular fractionation process used for the preparation of pellicles.

SDS-PAGE of the pellicle fractions of $T$. gondii showed the presence of several proteins ranging in size from 22, 30, 43, 50, 55, 65, 79, 90 and $160 \mathrm{kDa}$. This demonstrated that like most membranes, the $T$. gondii pellicle contains numerous proteins. The $30 \mathrm{kDa}$ protein is well known as the major surface antigen TgSAG1 (P30), whereas the $41 \mathrm{kDa}$ band is probably TgSAG3 (P43) or TgSRS2 (P43) respectively. Proteins at 22, 35 and $43 \mathrm{kDa}$, according to Foussard et al. (1990), correlate with antigens 
recognized by monoclonal antibodies to the $T$. gondii pellicle (Johnson, McDonald and Neoh, 1983; Kasper, Bradely and Pfefferkorn, 1984; Endo et al. 1988 ) and proteins in the $50-52 \mathrm{kDa}$ regions may be tubulin (Schwartzman et al. 1985). We did not confirm the identity of most of these proteins, as $T$. gondii was simply included in this study as a control for the methodology.

Subsequently, the plasmalemma was separated from the whole pellicle by high salt-glycerol treatment followed by 1 cycle of freezing/thawing and sonication. After removal of the undisrupted pellicles by centrifugation at $10000 \mathrm{~g}$, the supernatant was layered on top of a sucrose gradient and centrifuged at $140000 \boldsymbol{g}$ for $14 \mathrm{~h}$. The gradient contained a pellet and a very faint low-density band close to the top of the gradient. Electron microscopy showed this band contained membranous vesicles with diameters ranging from 20 to $100 \mathrm{~nm}$, which were also found distributed in all fractions throughout the gradient. Western blotting with antibody also confirmed the distribution of membrane containing antigen throughout the gradient and the presence of known surface antigens. Hence the evidence shows these vesicles are membranes containing surface molecules, namely plasmalemma. These results are therefore in keeping with those reported by Rabjeau et al. (1997) who developed this approach for isolating plasmalemma from $T$. gondii. It is of interest to note that our protein analyses of plasmalemma differ from those of Rabjeau et al. (1997) in that we find 2 abundant proteins in the plasmalemma preparations of T. gondii at 30 and $22 \mathrm{kDa}$. Fractions $10-12$ (Fig. 3a of Rabjeau et al. (1997) contain an abundant $30 \mathrm{kDa}$ antigen (SAG1) plus 2 or 3 other much lower abundance proteins (one of which migrates at approx. $20 \mathrm{kDa}$ ). We are unable to explain these differences in results, other than to note that a different laboratory stock of RH was used in this study compared to that used by Rabjeau et al. (1997).

Having established the methodology for preparing pellicles and plasmalemma from $T$. gondii, we applied this technology to the study of $N$. caninum. SDS-PAGE revealed at least 10 proteins being present in the $N$. caninum pellicle fractions at 29,32 , $38,40,50,55,65,70,110$ and $150 \mathrm{kDa}$ respectively. Some of these proteins have probably previously been identified by monoclonal antibodies raised to surface antigens (Hemphill et al. 1997; Björkman and Hemphill, 1998; Schares et al. 1999). Surface antigens of 30 and $32 \mathrm{kDa}$ of $N$. caninum were described by Björkman and Hemphill (1998); a $38 \mathrm{kDa}$ protein was identified as the major surface antigen NcSAG1 (P36) (Hemphill et al. 1997 a; Sonda et al. 1998 ) and a $43 \mathrm{kDa}$ surface protein was identified as a SRS2 homologue (Hemphill et al. 1997 b). Howe et al. (1998) described NcSAG1 and NcSRS2 as surface antigens of 29 and $36 \mathrm{kDa}$. Similar to
$T$. gondii, where the pellicle and plasmalemma contains an abundant amount of its major surface antigen SAG1 (P30), the results described here for $N$. caninum provide strong support for 2 abundant proteins in the plasmalemma of 30 and $22 \mathrm{kDa}$. This result was also obtained when these experiments were repeated with another strain of $N$. caninum (NcLiverpool, not shown).

The weight of evidence presented here leads us to conclude that the $30 \mathrm{kDa}$ antigen detected in the plasmalemma of $N$. caninum is the p29 described by Howe et al. (1998). We searched (using tblastn) the TIGR Gene Index database (http://www.tigr.org/ tigr-scripts/tgi/T_index.cgi? species=n_caninum) for sequences from $N$. caninum that possessed similarity to the SAG2 protein sequence of $T$. gondii. However, only a single EST was identified (CF967870) which possessed any similarity. The abundance of this EST (a singleton) suggests to us the $22 \mathrm{kDa}$ protein of $N$. caninum may not be a SAG2 homologue. The identity of these 2 proteins remains to be determined.

In conclusion, we provide further information, at the molecular level, on the protein composition of the pellicles and plasmalemmas of $T$. gondii and $N$. caninum. The results provided here show that the surface of the $N$. caninum tachyzoite contains 2 abundant proteins, as appears to be the case for $T$. gondii. Further studies on the role of these different proteins will help us understand how $N$. caninum interacts with host cells.

We are grateful to J. P. Dubey (USDA) for providing the Nc-1 isolate of $N$. caninum, J. Boothroyd (Stanford University) for DG 52, G. Schares for MAB 5.2.15 and 4.7.12 and S. Parmley for anti SAG2 antibody. We thank F. Foussard (Angers University, France) for expert advice throughout the course of this work, M. Jones (Queensland Institute of Medical Research, Australia) for his advice on electron microscopy during the early stages of this project, B. Booth (University of Technology, Sydney, Australia) for his assistance in ultracentrifugation. This research was performed in partial fulfillment for the degree of Ph.D. by Y.L.

\section{REFERENCES}

Björkman, C. and Hemphill, A. (1998). Characterisation of Neospora caninum iscom antigens using monoclonal antibodies. Parasite Immunology 20, 73-80.

Boothroyd, J. C., Hehl, A., Knoll, L. J. and

Manger, I. D. (1998). The surface of Toxoplasma: more and less. International Fournal for Parasitology 28, 3-9.

Couveur, G., Sadak, A., Fortier, B., and Dubremetz, J. F. (1988). Surface antigens of Toxoplasma gondii. Parasitology 97, 1-10.

Endo, T., Yagita, K., Yasuda, K., and Nakamura, T. (1988). Detection and localization of actin in Toxoplasma gondii. Parasitology Research 75, 102-106.

Foussard, F., Gallois, Y., Tronchin, G., Robert, R and Mauras, G. (1990). Isolation of the pellicle of 
Toxoplasma gondii (Protozoa, Coccidia) : characterization by electron microscopy and protein composition. Parasitology Research 76, 563-565.

Handman, E., Goding, J. W. and Remington, J. S. (1980). Detection and characterization of membrane antigens of Toxoplasma gondii. Fournal of Immunology 124, 2578-2583.

Harlow, E. and Lane, D. (1988). Immunoblotting. In Antibodies : a Laboratory Manual, pp. 490-492. Cold Spring Harbor Laboratory Press, Cold Spring Harbor.

Hemphill, A. (1996). Subcellular localization and functional characterization of Nc-P43, a major Neospora caninum tachyzoite surface protein. Infection and Immunity 64, 4279-4287.

Hemphill, A. and Gottstein, B. (1996). Identification of a major surface protein on Neospora caninum tachyzoites. Parasitology Research 82, 497-504.

Hemphill, A., Fuchs, N., Sonda, S., Gottstein, B. and Hentrich, B. (1997a). Identification and partial characterization of a $36 \mathrm{kDa}$ surface protein on Neospora caninum tachyzoites. Parasitology 115, 371-380.

Hemphill, A., Felleisen, R., Connolly, B., Gottstein, B., Hentrich, B. and Muller, N. (1997 b). Characterisation of a cDNA-clone encoding Nc-p43 a major Neospora caninum tachyzoite surface protein. Parasitology 115, 581-590.

Howe, D. K., Crawford, A. C., Lindsay, D. and Sibley, L. D. (1998). The P29 and P35 immunodominant antigens of Neospora caninum tachyzoites are homologous to the family of surface antigens of T. gondii. Infection and Immunity 66, 5322-5108

Jung, C., Lee, C. Y.-F. and Grigg, M. E. (2004). The SRS superfamily of Toxoplasma surface proteins. International Fournal for Parasitology 34, 285-296.

Johnson, A. M., McDonald, P. J. and Neoh, S. H. (1983). Monoclonal antibodies to Toxoplasma cell membrane surface antigens protect mice from Toxoplasmosis. Fournal of Protozoology 30, 351-357.

Kasper, L. H., Bradley, M. S. and Pfefferkorn, E. R. (1984). Identification of stage specific sporozoite antigens of Toxoplasma gondii by monoclonal antibodies. Fournal of Immunology 132, 443-449.

Mann, T. and Beckers, C. (2001). Characterisation of the subpellicle network, a filamentous membrane skeletal component in the parasite Toxoplasma gondii. Molecular and Biochemical Parasitology 115, 257-268.

Mauras, G., Laget, P. and Sennet, J. M. (1977). Isolation of Toxoplasma gondii membrane using latex microspheres. Biomedical Pharmacology 27, 3-4.

Morrissette, N. S., Bedian, V., Webster, P. and Roos, D. S. (1994). Charactersation of extreme apical antigens from Toxoplasma gondii. Experimental Parasitology 79, 445-459.
Nichols, B. A. and Chiappino, M. L. (1987). Cytoskeleton of Toxoplasma gondii. Fournal of Protozoology 34, 217-226.

Ogino, N. and Yoneda, C. (1966). The fine structure and mode of division of Toxoplasma gondii. Archives of Ophthamology 75, 218-227.

Porchet, E. and Torpier, G. (1977). Etude du germe infectieux de Sarcocystis tenella et Toxoplasma gondii par la techniquedu cryodecapage. Zeitschrift für Parasitenkunde 54, 101-124.

Rabjeau, A., Foussard, S., Mauras, G. and Dubremetz, J. F. (1997). Enrichment and biochemical characterization of Toxoplasma gondii tachyzoite plasmalemma. Parasitology 114, 421-426.

Schares, G., Dubremetz, J. F., Dubey, J. P., Barwald, A., Loyens, A. and Conraths, F. J. (1999). Neospora caninum: identification of $19-, 38-$, and $40-\mathrm{kDa}$ surface antigens and a 33-kda dense granule antigens using monoclonal antibodies. Experimental Parasitology 92, 109-119.

Schwartzman, J. D., Krug, E. C., Binder, L. I. and Payne, M. R. (1985). Detection of the microtubule cytoskeleton of the coccidian Toxoplasma gondii and the hemoflagellate Leishmania donovani by monoclonal antibodies specific for beta-tubulin. Fournal of Protozoology 32, 747-749.

Sheffield, H. G. and Melton, M. (1968). The fine structure and reproduction of Toxoplasma gondii. Fournal of Parasitology 54, 209-226.

Sonda, S., Fuchs, N., Connolly, B., Fernandez, P., Gottstein, B. and Hemphill, A. (1998). The major $36 \mathrm{kDa}$ Neospora caninum tachyzoite surface antigen protein is closely related to the major Toxoplasma gondii surface antigen. Molecular and Biochemical Parasitology 97, 97-108.

Sulzer, A., Strobel, P., Springer, E., Roth, I. and Callaway, C. (1974). A comparative electron microscopic study of the morphology of Toxoplasma gondii by freeze-etch replication and the in sectioning technique. Fournal of Protozology 21, 710-714.

Tryon, J. C. (1979). Toxoplasma gondii ultrastructure and antigenicity of purified tachyzoite pellicle. Experimental Parasitology 48, 198-205.

Vivier, E. and Petiprez, A. (1969). The outer membrane complex and its development at the time of the formation of daughter cells in Toxoplasma gondii. Fournal of Cell Biology 43, 329-342.

Vivier, E., Devauchelle, G., Petitprez, A., Porchet-Henere, E., Prensier, G., Schrevel, J. and Vinckier, D. (1970). Observations de cytology comparee chez les sporozoaries. 1. les structures superficielles chez les forms vegetatives. Protistologica 6 , 127-150. 